\title{
MEASUREMENT OF SPACE CHARGE EFFECT ON TRANSVERSE EMITTANCE
}

\author{
S. Cousineau*, K. Fung, M. Ball, C.M. Chu, V. Derenchuk, G. East, D. FrieselW. Guo, \\ A. Harbi, S.Y. Lee, T. Sloan, S. Wang, Y. Zhang, IUCF, Indiana University \\ J. Holmes ${ }^{\dagger}$ S. Shastry ${ }^{\ddagger}$, K.Y. Ng ${ }^{\S}$
}

\begin{abstract}
The SNS accumulator ring is designed to compress $1.5 \times 10^{14} 1 \mathrm{GeV}$ protons from a bunch train of $1000 \mu \mathrm{s} \mathrm{H}^{-}$ to a single proton bunch with bunch length $700 \mathrm{~ns}$. The injection scenario of the SNS is experimentally simulated at the Cooler Injector Synchrotron (CIS) at Indiana University. A $100 \mu \mathrm{s}, 7 \mathrm{MeV} H^{-}$bunch train from the RFQ and DTL is compressed into a single proton bunch with bunch length $\leq 150 \mathrm{~ns}$. Transverse rms emittance of the 7 $\mathrm{MeV}$ beam is measured by the quadrupole tuning method in the CIS extraction line. Measurements are taken at various beam intensities with peak currents ranging from a few $\mathrm{mA}$ to hundreds of $\mathrm{mA}$. Results are compared with simulation, and the effects of multiple strip foil transits and space charge are discussed.
\end{abstract}

\section{INTRODUCTION}

The Spallation Neutron Source currently under construction at Oak Ridge National Laboratory will deliver $1.5 \times 10^{14}, 1 \mathrm{GeV}$ protons per pulse to a liquid mercury target for neutron spallation at a frequency of $60 \mathrm{~Hz}$, thus making it the most intense source of neutrons in the world. One of the major challenges associated with building such a high intensity machine is to control and minimize radiation activation of the accelerator, an effect directly dependent on the fraction of particles which are lost inside the machine.

Due to its high intensity and low energy, a primary driving force for particle loss in the SNS will be space charge effects. The accumulator ring will accept a $1 \mathrm{~ms}$ pulse of protons from the linac and compress it to just under a $\mu \mathrm{s}$, thereby increasing the beam intensity by a factor of a thousand. Although extensive simulations have been performed to examine the consequences of this compression, little work has been dedicated to experimental investigation of the subject.

In an effort to fill the experimental deficit, we utilize the Cooler Injection Synchotron (CIS) at IUCF to mimic the injection and accumulation scenario of the SNS. Although the CIS maximum beam intensity is three orders of magnitude lower than SNS, the space charge effect is greater for lower energies and thus we can compensate for the difference in intensity by running the beam at a very low energy

\footnotetext{
* Work supported by an NSF grant, a DoE grant, and by the Spallation Neutron Source project, ORNL

${ }^{\dagger}$ SNS at ORNL

$\ddagger$ SUNY at Plattsburgh

$\S$ FNAL
}

(7 MeV), creating a space charge environment very similar to the SNS. We measure the emittance of the CIS beam as a function of accumulated beam intensity, and we compare our results with simulated data with and without space charge.

\section{EXPERIMENTAL PROCEDURE AND DATA ANALYSIS}

The Cooler Injection Synchotron at IUCF is composed of a $3 \mathrm{MeV}$ radio frequency quadrupole followed by a 7 $\mathrm{MeV}$ drift tube linac [1]. The $7 \mathrm{MeV} H^{-}$beam is injected into the synchotron through a chicane magnet and stripped into bare protons by a $3.6 \mu \mathrm{g} / \mathrm{cm}^{2}$ carbon foil. Under normal operating conditions, the beam would be adiabatically captured by the synchotron RF and ramped to 75-225 MeV before transport into the IUCF Cooler ring. However, for the purpose of this experiment, no adiabatic capture was performed and the beam was not accelerated beyond $7 \mathrm{MeV}$. Furthermore, to ensure clean extraction into the transfer line, the $\mathrm{H}^{-}$beam was chopped at the source to $83 \mathrm{~ns}$, a small fraction of the $477 \mathrm{~ns}$ period of the synchotron. A travelling wave kicker with a rise and fall-time of $35 \mathrm{~ns}$ and a flattop of $300 \mathrm{~ns}$ was used to extract the beam through a Lamberston septum magnet. The effective width of the septum is $11 \mathrm{~mm}$, and for a beam with momentum spread on the order of $.1 \%$, the maximum horizontal emittance that can clear the septum is about $9.6 \pi \mathrm{mm} \cdot \mathrm{mrad}$.

Upon extraction from the synchotron, the beam enters the transfer line to the Cooler, where it passes through two quadrupoles separated by 20 inches. A harp outfitted with fifty wires spaced $.5 \mathrm{~mm}$ apart was placed 16 inches downstream from the second quadropole and used to measure the beam profile. Beam emittances were derived via the quadrupole tuning method, which utilizes the relationship between quadrupole strength and rms beam size [2]. The emittance measured by this method includes the effect of dispersion at the harp, and in order to find the true emittance we must correct for this difference. An analytical account of the emittance measurement procedure as well as the dispersion subtraction is easily derived. First note that the dispersion function and the betatron phase space coordinates obey the transport equations,

$$
\left(\begin{array}{c}
D \\
D^{\prime} \\
1
\end{array}\right)_{2}=\left(\begin{array}{cc}
M & \bar{d} \\
0 & 1
\end{array}\right)_{21}\left(\begin{array}{c}
D \\
D^{\prime} \\
1
\end{array}\right)_{1}
$$




$$
\left(\begin{array}{c}
x_{\beta} \\
x_{\beta}^{\prime} \\
1
\end{array}\right)_{2}=\left(\begin{array}{cc}
M & \bar{d} \\
0 & 1
\end{array}\right)_{21}\left(\begin{array}{c}
x_{\beta} \\
x_{\beta}^{\prime} \\
1
\end{array}\right)_{1}
$$

where $M$ is a $2 \times 2$ matrix, and $\bar{d}$ is a $2 \times 1$ dispersion vector. Since the transverse displacement from the closed orbit is given by $x=x_{\beta}+D \delta$ and $x^{\prime}=x_{\beta}^{\prime}+D^{\prime} \delta$, where $\delta$ is the fractional off-momentum deviation, we obtain

$$
\left(\begin{array}{c}
x \\
x^{\prime} \\
1
\end{array}\right)_{2}=\left(\begin{array}{cc}
M & \bar{d} \\
0 & 1
\end{array}\right)_{21}\left(\begin{array}{c}
x \\
x^{\prime} \\
1
\end{array}\right)_{1} .
$$

Let $\mathbf{x}=\left(x, x^{\prime}, \delta\right)$ be the transpose of the state vector $\mathbf{x}$. We define the generalized $\sigma$ matrix as

$$
\sigma=\langle(\mathbf{x}-\langle\mathbf{x}\rangle)(\mathbf{x}-\langle\mathbf{x}\rangle) \dagger\rangle
$$

where $\langle\ldots\rangle$ stands for the average of a physical quantity over the distrbution function. The transport of the generalized $\sigma$-matrix through accelerator elements is

$$
\sigma\left(s_{2}\right)==\left(\begin{array}{cc}
M & \bar{d} \\
0 & 1
\end{array}\right)_{21} \sigma\left(s_{1}\right)\left(\begin{array}{cc}
M & \bar{d} \\
0 & 1
\end{array}\right)_{21}^{\dagger}
$$

In our experiments, there is no dipole between $s_{1}$ and $s_{2}$, so $\bar{d}=0$, and thus

$$
\sigma_{11}\left(s_{2}\right)=M_{11}^{2} \sigma_{11}\left(s_{1}\right)+M_{12}^{2} \sigma_{22}\left(s_{1}\right)+2 M_{11} M_{12} \sigma_{12}\left(s_{1}\right) .
$$

Since we have determined $\sigma_{11}$ by varying quadrupole field, i.e. varying $M_{i j}$ 's (quadrupole tuning method), we obtain the coefficients of the sigma matrix elements at $s_{1}$. The rms emittance of the beam is then given by

$$
\begin{aligned}
\epsilon_{\mathrm{rms}}^{2} & =\sigma_{x_{\beta}}^{2} \sigma_{x_{\beta}^{\prime}}^{2}-\sigma_{x_{\beta} x_{\beta}^{\prime}}^{2} \\
& =\left(\sigma_{11}-D^{2} \sigma_{\delta}^{2}\right)\left(\sigma_{22}-D^{\prime 2} \sigma_{\delta}^{2}\right) \\
& -\left(\sigma_{12}-D D^{\prime} \sigma_{\delta}^{2}\right)^{2}
\end{aligned}
$$

Here we have assumed that $\langle x\rangle=0,\left\langle x^{\prime}\right\rangle=0$, and $\langle\delta\rangle=0$ for a symmetric beam distribution.

The basic experimental procedure consisted of accumulating the chopped beam pulses in the synchotron for a set number of turns and then extracting the beam onto the harp for emittance measurement. In an effort to obtain statistically sound data, five profile measuremets were recorded for each quadrupole setting, and at least twenty quadrupole settings were applied to every turn iteration. Both vertical and horizontal emittances were measured every tenth turn beginning with turn 10 and ending with turn 200, when CIS begins to lose the beam.

The current measured by two different sources along the DTL indicated that the number of protons entering CIS during each pulse was $\sim 10^{9}$. The CIS saturation limit typically occurs at around $10^{11}$ particles, so we were able to inject about 100 turns before we reached this limit. The harp profiles are found to be well approximated by a gaussian distribution function, and we derive the rms beam widths by numerical fits to the noise-subtracted data. Figure 1 shows a plot of one of these fits for a horizontal profile of the beam after 50 accumulation turns.

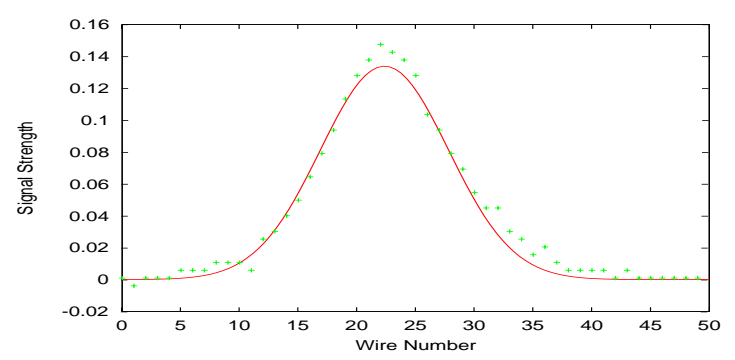

Figure 1: Horizontal beam profile on the harp after 50 accumulation turns and the numerical fit to the data.

The errorbars on the beam widths are conservatively taken to be $\pm .25 \mathrm{~mm}$, the wire spacing in the harp, and are propogated through the analysis. The beam widths are plotted against the quadrupole strengths and a parabolic fit to the data gives the final emittance. Figure 2 shows the parabola for the 50 turn case with its numerical fit.

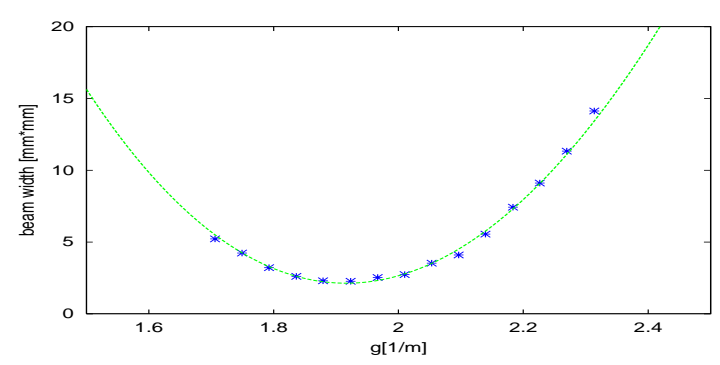

Figure 2: Horizontal beam widths versus quad. strength after 50 accumulations turns and the numerical fit to the data.

We unfortunately find that the close proximity of the harp to the vertical quadrupole complicates the analysis of vertical data and ultimately renders the emittance measurement meaningless. The vertically focused beam covers only a few harp wires, so an accurate fit of the beam width is difficult if not impossible to obtain. In addition, the beam is very sensitive to quadrupole strength at this location and the range of available strengths is very small, i.e., the parabola is too thin and thus the $\sigma$ parameters can not be well constrained. Fortunately, the horizontal profile at this location is wide and the quadrupole range large, so we focus our attentions on this plain instead.

The results of the horizontal emittance versus intensity measurement are discussed in the following section, along with comparisons of simulated and measured data.

\section{SIMULATION AND DISCUSSION}

A good measure of the strength of the space charge effect in a machine is the inchoherent space charge tune shift. The 
SNS machine is expected to have $\Delta \nu_{s c} \approx .15-.2$ and CIS running at $7 \mathrm{MeV}$ also has $\Delta \nu_{s c} \approx .2$. Although the machine lattices differ greatly, the space charge physics within each machine should be comprable. Emittance growth for the CIS beam can be accounted for in large part by two mechanisms: scattering and energy loss at the strip foil, and space charge halo growth. Whereas the effect of space charge on the beam can be somewhat elusive, the strip foil mechanism is well understood and we can predict its contribution to the emittance growth; any remaining emittance growth we associate with space charge. In our simulations using ORBIT, a particle-in-cell simulation code [3], we model strip foil scattering with a repeated single scatterers routine.

A comparison between the measured data and the simulated data is shown in Figure 3. Included in the plot are the results of a simulation with strip-foil effects alone, and the results of another simulation with both space charge and strip foil effects, using ORBIT's Green's function model of space charge. Neither result agrees exactly with the experimental data, but it is obvious that before saturation the strip-foil alone grossly underestimates the amount of emittance growth, while the inclusion of space charge compensates for most of this deficit.

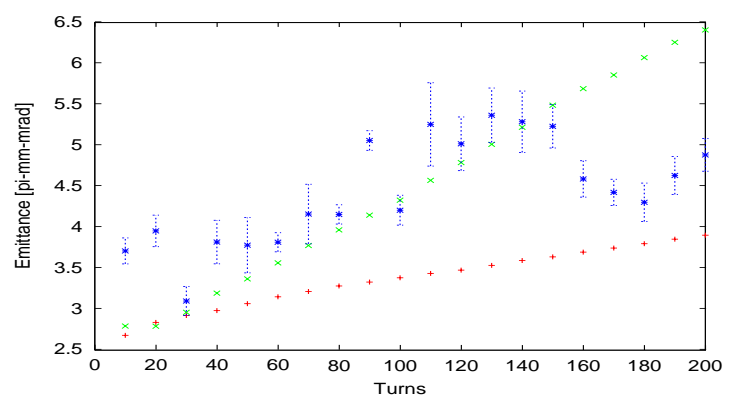

Figure 3: Measured and simulated emittances versus turn. The +'s are simulation with foil only, and x's are simulation with foil and space charge.

In anticipation of future space charge experiments with the CIS ring, simulations were performed with injection intensities an order of magnitude greater than currently available to the ring. At this intensity the space charge tune shift of the beam is increased to about $\Delta \nu_{s c} \approx .25$, and we begin to see halo develop in the vertical phase space. Figures 4 and 5 show the vertical phase space after 50 turns with the current injection intensity and the intensity one order of magnitude higher, respectively. The second moment of the beam, shown in Figure 6, undergoes a half-oscillation during every turn, hinting strongly that the halo is the result of a half-integer envelope resonance. This idea is currently under investigation.

\section{CONCLUSIONS AND FUTURE WORK}

We have successfully mimicked the accumulation scenario of the SNS compressor ring with the CIS booster

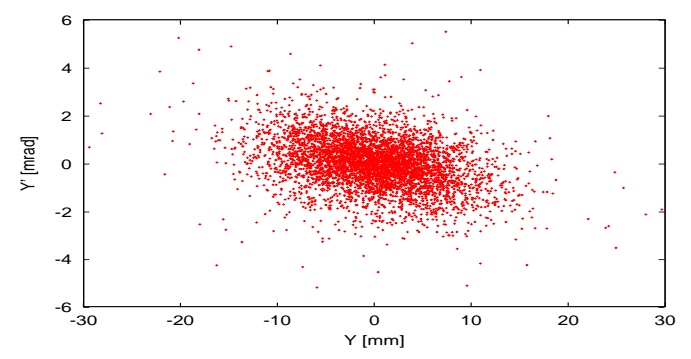

Figure 4: Vertical phase space distribution after 50 accumulation turns, injecting $1 \times 10^{9}$ particles per turn.

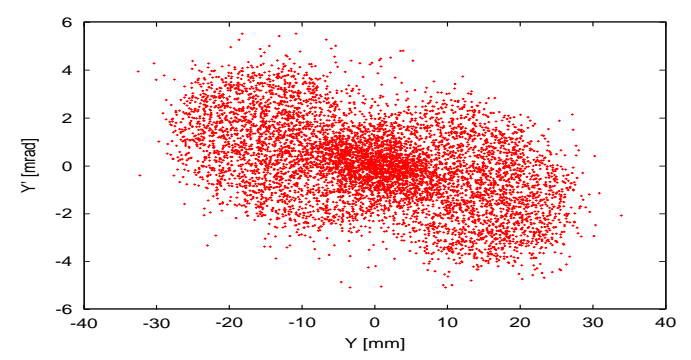

Figure 5: Vertical phase space distribution after 50 accumulation turns, injecting $1 \times 10^{10}$ particles per turn.

ring at Indiana University, and measured emittance evolution as a function of accumulated beam intensity. We see from comparisons of experimental data with simulated data that space charge plays an important role in the emittance growth of the beam. In addition, simulations indicate that an envelope resonance develops in the beam at intensities an order of magnitude higher than what was injected in the experiment. Plans for further work in this area include investigating the threshold intensity and nature of this resonance, as well as improving trasmission of the CIS beam through the RFQ in hopes of achieving this threshold intensity and experimentally verify the resonance.

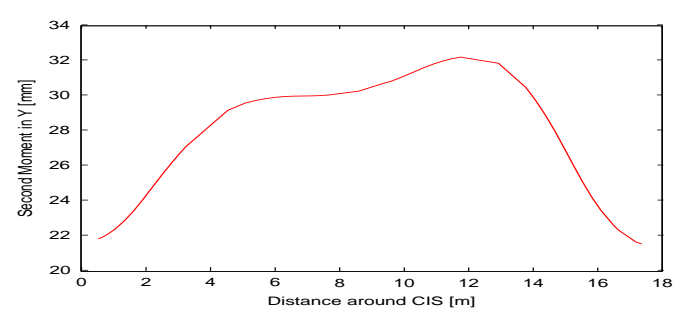

Figure 6: Second moment of the beam over one turn (turn $50)$.

\section{REFERENCES}

[1] X. Kang, The Cooler Injector Synchotron at IUCF, Ph.D. thesis, Indiana University, 1999, unpublished.

[2] See e.g. S.Y Lee Accelerator Physics, p. 55 (World Scientific, 1999)

[3] J. Galambos et al., ORBIT User's Manual, SNS/ORNL/AP Technical Note 011, 1999. 